\title{
Robust Adaptation and Homeostasis by Autocatalysis
}

\author{
T. Drengstig ${ }^{\dagger}$ X. Y. Ni, ${ }^{\ddagger}$ K. Thorsen, ${ }^{\dagger}$ I. W. Jolma, ${ }^{\ddagger}$ and P. Ruoff* ${ }^{*}+$ \\ ${ }^{\dagger}$ Department of Electrical Engineering and Computer Science, ${ }^{\ddagger}$ Centre for Organelle Research, University of Stavanger, N-4036 \\ Stavanger, Norway
}

ABSTRACT: Robust homeostatic mechanisms are essential for the protection and adaptation of organisms in a changing and challenging environment. Integral feedback is a control-engineering concept that leads to robust, i.e., perturbation-independent, adaptation and homeostatic behavior in the controlled variable. Addressing two-component negative feedback loops of a controlled variable $A$ and a controller molecule $E$, we have shown that integral control is closely related to the presence of zero-order fluxes in the removal of the manipulated variable $E$.

Here we show that autocatalysis is an alternative mechanism to obtain integral control. Although the conservative and marginal stability of the Lotka-Volterra oscillator (LVO) with autocatalysis in both $A$ and $E$ is often considered as a major inadequacy, homeostasis in the average concentrations of both $A$ and $E(\langle A\rangle$ and $\langle E\rangle)$ is observed. Thus, autocatalysis does not only represent a mere driving force, but may also have regulatory roles.

\section{INTRODUCTION}

Living organisms have the remarkable property to adapt to external environmental changes by keeping their "internal environment" at an approximately constant level. ${ }^{1}$ The development of the concept of homeostasis, i.e., the presence of coordinated physiological processes that maintain internal stability in organisms is attributed to Cannon, who also coined the term homeostasis during the 1920s.,

After Cannon, the concept of homeostasis broadened and other terminologies were introduced, either related to (circadian) set-point changes as in predictive homeostasis ${ }^{4}$ and rheostasis, ${ }^{5}$ or, as for the concept of allostasis, ${ }^{6,7}$ by considering both behavioral and physiological processes that maintain internal parameters within certain essential limits.

During the 1920s, Lotka ${ }^{8}$ investigated the physicochemical basis of homeostasis by considering the principle of Le Chatelier. The principle states that upon an external disturbance a chemical system in equilibrium will change to that direction, which minimizes the external disturbance. ${ }^{9}$ Lotka rejected the principle as a basis for homeostatic behavior and made a clear distinction between an organism's steady state and chemical equilibrium.

With the developments within control and systems theory, $^{10-12}$ the description of homeostatic behavior by feedback regulation came into focus ${ }^{13-15}$ with recent emphases on reaction kinetic and genetic models and network motifs. $^{16-23}$

Some of the mechanisms that account for perfect adaptation or homeostasis, including temperature compensation, ${ }^{24}$ are based on a balance between various opposing components within a reaction network. ${ }^{25}$

While a balancing-based approach does not guarantee a fixed steady state of the controlled variable in the presence of perturbations, the question arose how robust, i.e., perturbation- independent, homeostatic mechanisms could be achieved. From a control-engineering aspect integral control can keep systems at a given set-point even under the presence of uncontrollable perturbations.

Figure 1a illustrates the concept of integral control, where $A$ is the controlled variable with set-point $A_{\text {set }}$. The integral controller is embedded within a negative feedback loop, which is characterized by defining the error $e$ between $A$ and $A_{\text {set }}$ as ${ }^{26}$

$$
e=A_{\text {set }}-A
$$

The controller integrates the error $e$ over time, which results in the manipulated variable $E$

$$
E(t)=K_{i} \int_{0}^{t} e\left(t^{\prime}\right) \mathrm{d} t^{\prime}
$$

where $K_{i}$ is a constant called the (integral) gain of the controller. $^{26}$ The variable $E$ then feeds into the process that generates $A$ and adjusts the level of $A$ in the presence of (unpredictable) environmental perturbations. The advantage of integral control is that the steady state value of $A$ will approach, without error, the set-value $A_{\text {set }}$. For a formal proof, see, for example, ref 26 . With respect to applying integral control to the regulation of cellular and biochemical processes the question arises how error sensing mechanisms can be achieved in reaction kinetic terms.

The implementation of integral control to reaction kinetic networks was emphasized by Yi et al. ${ }^{16}$ and others. ${ }^{22,23,27} \mathrm{We}$ have recently shown that zero-order kinetics in the removal of a controller variable within negative feedback loops is a necessary condition to obtain integral control. ${ }^{21}$ The principle is

Received: January 14, 2012

Revised: April 10, 2012

Published: April 16, 2012 


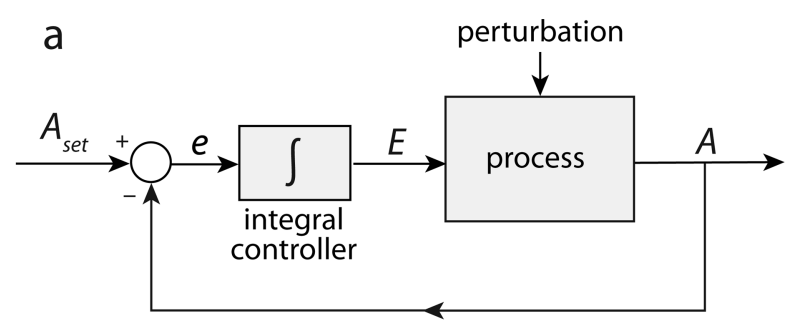

b

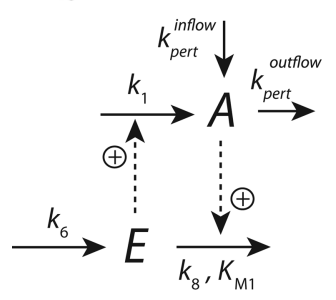

$$
\begin{aligned}
\dot{A} & =k_{\text {pert }}^{\text {inflow }}-k_{\text {pert }}^{\text {outlow }} \cdot A+k_{1} \cdot E \\
\dot{E} & =k_{6}-\frac{k_{8} \cdot E}{\mathrm{~K}_{\mathrm{M} 1}+E} \cdot A \\
& \Downarrow \mathrm{k}_{\mathrm{M} 1} \ll E \\
\dot{\mathrm{E}}= & \mathrm{k}_{8}\left(\frac{\mathrm{k}_{6}}{\mathrm{k}_{8}}-A\right)=\mathrm{k}_{8}(\underbrace{A_{\text {set }}^{\text {in }}-A}_{\mathrm{e}})
\end{aligned}
$$

C

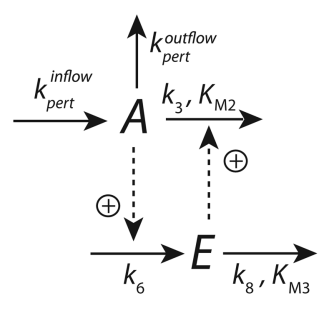

$$
\begin{aligned}
\dot{A}= & k_{\text {pert }}^{\text {inflow }}-k_{\text {pert }}^{\text {outflow }} \cdot A-\frac{k_{3} \cdot A}{k_{M 2}+A} \cdot E \\
\dot{E}= & k_{6} \cdot A-\frac{k_{8} \cdot E}{k_{M 3}+E} \\
& \Downarrow k_{M 3} \ll E \\
\dot{E}= & -k_{6}\left(\frac{k_{8}}{k_{6}}-A\right)=-k_{6}(\underbrace{A_{\text {set }}^{\text {out }}-A}_{e})
\end{aligned}
$$

Figure 1. Integral control by zero-order kinetics. (a) Scheme of the integral control concept. $A$ is the controlled variable, which is regulated to set-point $A_{\text {set }}$ regardless of unpredictable perturbations of $A$. To achieve this, the error $e=A_{\text {set }}-A$ is calculated and integrated leading to the manipulated variable $E$, which corrects the value of $A$ such that $A$ will approach $A_{\text {set }}$ (b) Negative feedback loop showing robust homeostasis in $A$ when the manipulated variable $E$ is removed by zero-order kinetics. In this case $\dot{E}$ is proportional to the error $e$, which, when integrated, leads to $E$ and adjusts the level of $A$ precisely to its set-point $A_{\text {set }}^{i n}$. Because the controller action is based on adding $A$ by $E$, we term this controller type for an inflow controller. (c) Kinetic representation of an outflow controller with integral control by zeroorder removal of $E$.

illustrated in parts $\mathrm{b}$ and $\mathrm{c}$ of Figures 1 on two of eight ${ }^{28}$ possible two-component molecular controller/network motifs (negative feedback loops), where $A$ is the (homeostatic) controlled variable with set-point $A_{\text {set }}$, and $E$ is the manipulated variable. As indicated in the general scheme (Figure 1a), the concentration of $E$ in the two network motifs (Figures $1 \mathrm{~b}$ and c) is proportional to the error between $A$ and its set-point $A_{\text {set }}$. Because the controller action in Figure $1 \mathrm{~b}$ is based on a $E$ mediated addition of $A$, we term this type of controller motif for an inflow controller. It may be noted that the homeostatic performance of inflow controllers breaks down when an uncontrolled inflow perturbation of $A$ becomes larger than the consumption of $A^{21}$ in the system.

In Figure 1c, the representation of an outflow controller is shown. In outflow controllers robust homeostasis of $A$ at $A_{\text {set }}$ can be achieved by an E-mediated removal of excess $A$. As in case of the inflow controller, integral control occurs when $E$ is formed and removed by zero-order kinetics. ${ }^{21}$ Also here it may be noted that homeostasis by outflow controllers is lost when an uncontrolled outflow of $A$ from the system dominates over the regular inflow of $A$ into the system. The combination of inflow and outflow controllers enables robust homeostasis by allowing to integrate processes such as environmentally dependent uptake and assimilation of $A$, its excretion, storage, as well as remobilization from a store. ${ }^{28,29}$

In the following, we show that the requirement of zero-order kinetics to achieve integral control can be replaced by autocatalysis.

\section{COMPUTATIONAL METHODS}

Computations were performed in parallel using the Fortran subroutine LSODE ${ }^{30}$ and MATLAB/SIMULINK (mathwork.com) together with the program PPLANE. ${ }^{31}$ Absoft's Pro Fortran compiler (absoft.com) was used together with the random number generator RAN1 described by Press et al. ${ }^{32}$ To make notations simpler, concentrations of compounds are denoted by compound names without square brackets. Concentrations and rate constants are given in arbitrary units (a.u.).

\section{INTEGRAL CONTROL BY AUTOCATALYSIS}

Parts $\mathrm{a}$ and $\mathrm{b}$ of Figure 2 show the negative feedback loops of the inflow and outflow controllers from Figure 1, respectively, but instead of using zero-order degradation of $E, E$ is formed autocatalytically and the degradation with respect to $E$ is firstorder. Because of the autocatalysis, $\mathrm{d}[\ln (E)] / \mathrm{d} t$ is now proportional to the error $e$ between the level of $A$ and its setpoint $A_{\text {set }}$ (Figure 2). Parts c and d of Figure 2 show the steady state values of $A\left(A_{s s}\right)$ for the inflow and outflow controller, respectively, at different inflow and outflow fluxes to and from $A$ described by the varying rate constants $k_{\text {pert }}^{\text {inflow }}$ and $k_{\text {pert }}^{\text {outlow }}$, respectively. Both show the typical behavior for inflow and outflow controllers. ${ }^{28}$ For the inflow controller, homeostasis breaks down (i.e., state steady values of $A$ increase above the set-point) when $k_{\text {pert }}^{\text {inflow }}$ dominates over the outflow fluxes (Figure 2c). For the outflow controller, the homeostatic behavior breaks down (i.e., state steady values of $A$ decrease below the set-point) when $k_{\text {pert }}^{\text {ouflow }}$ becomes large relative to the inflow fluxes (Figure 2d).

The steady state solutions for $A$ and $E$ of the inflow and outflow controllers in Figure 2 show either stable nodes or stable focus points (with or without saddle points), depending on the rate constants; see Figure 3. As an example, we consider the inflow controller in Figure 2a, which has two steady state solutions:

$$
\begin{aligned}
& \text { 1: } A_{s s}=k_{\text {pert }}^{\text {inflow }} / k_{\text {pert }}^{\text {outflow }}, \quad E_{s s}=0 \\
& 2: A_{s s}=k_{6} / k_{8}, \quad E_{s s}=\frac{k_{\text {pert }}^{\text {ouflow }} k_{6}-k_{\text {pert }}^{\text {inflow }} k_{8}}{k_{1} k_{8}}
\end{aligned}
$$

In case $k_{\text {pert }}^{\text {inflow }} \leq k_{\text {pert }}^{\text {outflow }} A_{\text {set }}^{\text {in }}$ homeostasis is preserved and eq 3 corresponds to a saddle point (green dots in Figure 3, parts a and c), whereas eq 4 corresponds to either a stable node (Figure 3a) or a stable focus (Figure 3c), determined by the eigenvalues of the system ${ }^{33}$

$$
\lambda_{1,2}=\frac{-k_{\text {pert }}^{\text {outflow }} \pm \sqrt{k_{\text {pert }}^{\text {oufflow }}{ }^{2}-4 k_{6} k_{\text {pert }}^{\text {ouflow }}+4 k_{\text {pert }}^{\text {inflow }} k_{8}}}{2}
$$

In case $k_{\text {pert }}^{\text {inflow }}>k_{\text {pert }}^{\text {outlow }} A_{\text {set }}^{\text {in }}$ homeostasis breaks down, and the only physical realistic steady state solution is eq 3 (Figure $3 \mathrm{~b}$ ). 


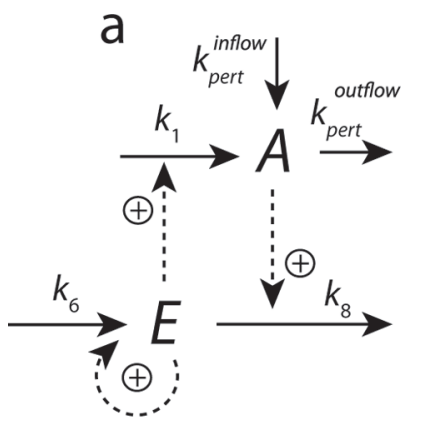

$$
\begin{aligned}
\dot{A} & =k_{\text {pert }}^{\text {inflow }}-k_{\text {pert }}^{\text {outflow }} \cdot A+k_{1} \cdot E \\
\dot{E} & =k_{6} \cdot E-k_{8} \cdot E \cdot A \\
& =E\left(k_{6}-k_{8} \cdot A\right) \\
\Downarrow & k^{\dot{n}(E)}=k_{6}-k_{8} \cdot A=k_{8}(\underbrace{A_{\text {set }}^{\text {in }}-A}_{e})
\end{aligned}
$$

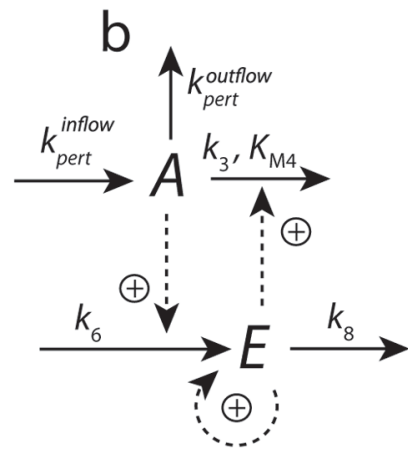

$$
\begin{aligned}
\dot{A} & =k_{\text {pert }}^{\text {inflow }}-k_{\text {pert }}^{\text {outflow }} \cdot A-\frac{k_{3} \cdot A}{k_{M 4}+A} \cdot E \\
\dot{E} & =k_{6} \cdot A \cdot E-k_{8} \cdot E \\
& =E\left(k_{6} \cdot A-k_{8}\right) \\
& \Downarrow \\
\ln (E) & =k_{6} \cdot A-k_{8}=-k_{6}(\underbrace{A_{\text {set }}^{\text {out }}-A})
\end{aligned}
$$
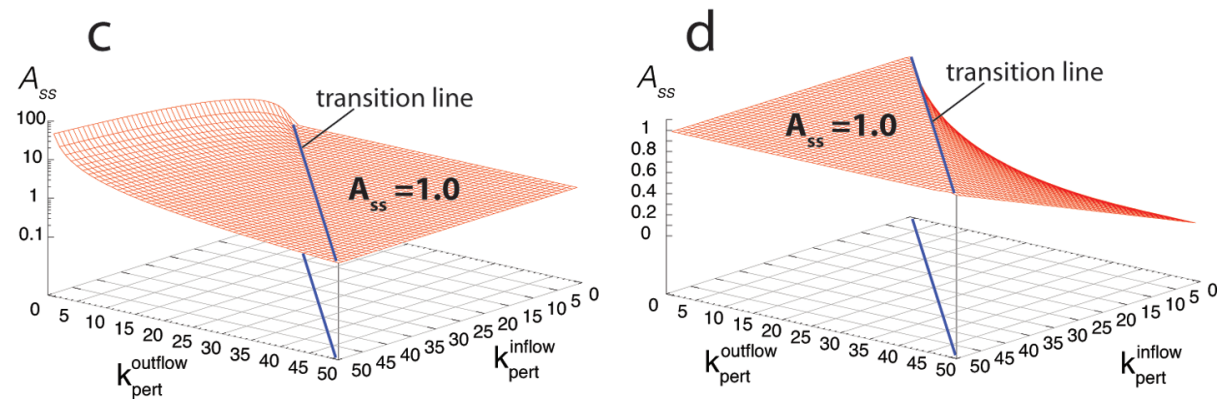

Figure 2. Integral control by autocatalysis. (a) Negative feedback loop of inflow controller with autocatalytic loop in $E$. $\mathrm{d}[\ln (E)] / \mathrm{d} t$ is proportional to the error $e$ between $A$ and its set-point $A_{\text {set }}^{\text {in }}=k_{6} / k_{8}$. (b) Negative feedback loop of outflow controller with autocatalytic loop in $E$. d $[\ln (E)] / \mathrm{d} t$ is proportional to the error $e$ between $A$ and its set-point $A_{\text {set }}^{\text {out }}=k_{8} / k_{6}$. (c) Homeostatic behavior of inflow controller described in (a). Rate constants $k_{\text {pert }}^{\text {inflow }}$ and $k_{\text {pert }}^{\text {outflow }}$ are allowed to vary between 0.5 and 50.0 with intervals by 0.5 , while all other rate constants are kept at 1.0. Homeostasis in $A$ steady state levels $\left(A_{s s}\right)$ with $A_{\text {set }}^{\text {in }}=1.0$ is observed when $k_{\text {pert }}^{\text {inflow }} \leq A_{\text {set }}^{\text {in }} k_{\text {pert }}^{\text {oufflow }}$, but lost when $k_{\text {pert }}^{\text {inflow }}>A_{\text {see }}^{\text {in }} k_{\text {pert }}^{\text {oufflow }} .{ }^{28}$ The transition line $k_{\text {pert }}^{\text {inflow }}=A_{\text {set }}^{\text {in }}$ pert ouflow $_{\text {separating }}$ homeostatic and nonhomeostatic regimes is indicated in blue. (d) Homeostatic behavior of outflow controller. Rate constants are allowed to vary as in part c, while the other rate constants are kept at 1.0. Homeostasis in $A$ steady state levels $\left(A_{\text {set }}^{\text {out }}=1.0\right)$ is observed when $k_{\text {pert }}^{\text {inflow }} \geq A_{\text {set }}^{\text {out }} k_{\text {pert }}^{\text {oufflow }}$, but lost when $k_{\text {pert }}^{\text {inflow }}<A_{\text {set }}^{\text {out }} k_{\text {pert }}^{\text {outflow }}$.

Hence, the manipulated variable $E$ becomes zero, and the steady state level of $A$ is determined by the relationship of the perturbation fluxes. Similar results are shown for the outflow controller (Figures 3d-f).

\section{CONSERVATIVE OSCILLATIONS}

By neglecting outflow perturbation $k_{\text {pert }}^{\text {outflow }}$ in the dynamics of $A$, the rate equations for the outflow controller in Figure $2 b$ becomes

$$
\begin{aligned}
& \dot{A}=k_{\text {pert }}^{\text {inflow }}-\frac{k_{3} A E}{K_{M 4}+A} \\
& \dot{E}=k_{6} A E-k_{8} E
\end{aligned}
$$

Moreover, when $K_{M 4} \rightarrow 0$ and making the substitution $\xi=$ $\ln (E)$, eqs 6 and 7 can be expressed as follows:

$$
\begin{aligned}
& \dot{A}=k_{\text {pert }}^{\text {inflow }}-k_{3} e^{\xi} \\
& \dot{\xi}=k_{6} A-k_{8}
\end{aligned}
$$

From eqs 8 and 9, an "energy-function" (H-function) can be constructed

$$
H(A, \xi)=-\int \dot{A} \mathrm{~d} \xi+\int \dot{\xi} \mathrm{d} A
$$

satisfying the equations

$$
\frac{\partial H}{\partial \xi}=-\dot{A} \quad \frac{\partial H}{\partial A}=\dot{\xi}
$$

showing that $H$ is time independent and the system is conservative 

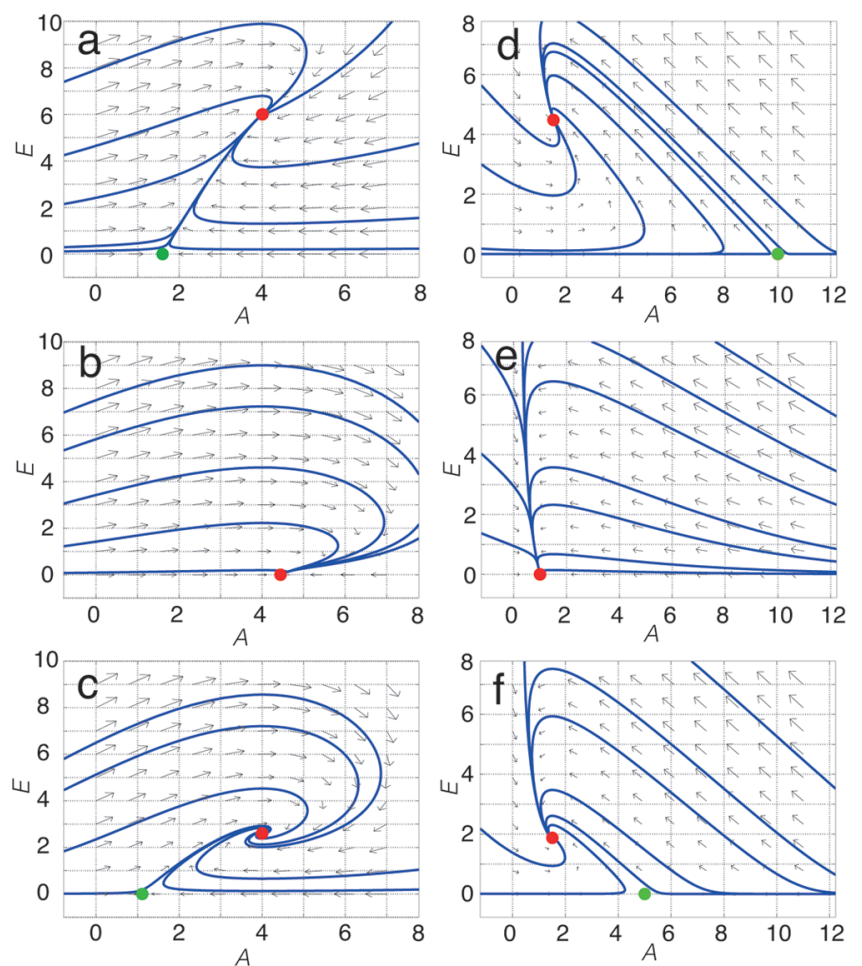

Figure 3. The inflow and outflow controllers can show stable nodes or stable focus points (with or without saddle points) depending on the inflow and outflow perturbations. Panels a-c: inflow controller from Figure 2a with $k_{1}=2.0, k_{6}=2.0, k_{8}=0.5$. (a) $k_{\text {pert }}^{\text {inflow }}=8.0$ and $k_{\text {pert }}^{\text {outlow }}=$ 5.0 showing stable node (red dot) at $A_{s s}=A_{s e t}^{i n}=k_{6} / k_{8}=4.0$ and $E_{s s}=$ 6.0 (eq 4). Green dot indicates a saddle point at $A_{s s}=k_{\text {pert }}^{\text {inflow }} / k_{\text {pert }}^{\text {ouffow }}=$ 1.6 and $E_{s s}=0$ (eq 3). Homeostasis in $A$ is preserved. (b) Example of controller breakdown when $k_{\text {pert }}^{\text {inflow }}=8.0$ and $k_{\text {pert }}^{\text {ouflow }}=1.8$ showing a stable node (red dot) at $A_{s s}=k_{\text {pert }}^{\text {inflow }} / k_{\text {pert }}^{\text {ouflow }}=4.44$ and $E_{s s}=0$ (eq 3). Homeostasis in $A$ is not preserved. The solution from eq 4 gives negative and therefore unrealistic $E_{s s}$ values. (c) $k_{\text {pert }}^{\text {inflow }}=2.0$ and $k_{\text {pert }}^{\text {ouffow }}$ $=1.8$ showing stable focus point (red dot) at $A_{s s}=A_{s e t}^{i n}=4.0$ and $E_{s s}=$ 2.6 (eq 4). Homeostasis in $A$ is preserved. Green dot indicates a saddle point at $A_{s s}=k_{\text {pert }}^{\text {inflow }} / k_{\text {pert }}^{\text {oufflow }}=1.11$ and $E_{s s}=0$ (eq 3). Panels d-f: outflow controller from Figure $2 \mathrm{~b}$ with $k_{3}=1.0, k_{6}=1.0, k_{8}=1.5$. (d) $k_{\text {pert }}^{\text {inflow }}=8.0$ and $k_{\text {pert }}^{\text {outlow }}=0.8$ showing stable node $($ red dot $)$ at $A_{s s}=A_{\text {set }}^{\text {out }}$ $=k_{8} / k_{6}=1.5$ and $E_{s s}=4.6$. Green dot indicates a saddle point at $A_{s s}=$ $k_{\text {pert }}^{\text {inflow }} / k_{\text {pert }}^{\text {outflow }}=10.0$ and $E_{s s}=0$. Homeostasis in $A$ is preserved. (e) Example of controller breakdown when $k_{\text {pert }}^{\text {inflow }}=4.0$ and $k_{\text {pert }}^{\text {oufflow }}=4.0$ showing a stable node (red dot) at $A_{s s}=k_{\text {pert }}^{\text {infl }} / k_{\text {pert }}^{\text {ouflow }}=1.0$ and $E_{s s}=0$. Homeostasis in $A$ is not preserved. (f) $k_{\text {pert }}^{\text {inflow }}=4.0$ and $k_{\text {pert }}^{\text {outflow }}=0.8$ showing stable focus point (red dot) at $A_{s s}=A_{s e t}^{o u t}=1.5$ and $E_{s s}=1.9$. Homeostasis in $A$ is preserved. Green dot indicates a saddle point at $A_{s s}=k_{\text {pert }}^{\text {inflow }} / k_{\text {pert }}^{\text {oufflow }}=5.0$ and $E_{s s}=0$.

$$
\frac{\partial H}{\partial t}=\frac{\partial H}{\partial A} \dot{A}+\frac{\partial H}{\partial \xi} \dot{\xi}=-\frac{\partial H}{\partial A} \frac{\partial H}{\partial \xi}+\frac{\partial H}{\partial \xi} \frac{\partial H}{\partial A}=0
$$

When $K_{M 4} \rightarrow 0$ the trajectories of the system (eqs 8 and 9) become closed orbits at constant values of $H$. Figure 4a shows the numerically calculated trajectory of a closed orbit at a relative low $K_{M 4}$ value. The same orbit as in Figure $4 \mathrm{a}$ is obtained in Figure $4 \mathrm{~b}$ (blue curve) by calculating the $\mathrm{H}$ function and finding the contour line at $H=-5.65 \mathrm{au}$, which is determined by the initial conditions for $A$ and $E$ in Figure 4a.

Despite its oscillatory character the system still shows homeostasis in $A$, but now for the average value of $A$, defined as

$$
\langle A\rangle=\frac{1}{\tau} \int_{0}^{\tau} A(t) \mathrm{d} t
$$

with $\langle A\rangle=A_{\text {set }}^{\text {out }}=k_{8} / k_{6}$ and $\tau$ as the simulation (integration) time, i.e., $\langle A\rangle$ is independent of $k_{\text {pert }}^{\text {inflow }}$ and $k_{3}$.

The independence of $\langle A\rangle$ from $k_{\text {pert }}^{\text {inflow }}$ is illustrated in Figures $4 \mathrm{c}$ and $4 \mathrm{~d}$, where $k_{\text {pert }}^{\text {inflow }}$ is successively increased. While a change in the amplitude in $A$ is observed, the change is symmetrical around $A_{\text {set }}^{\text {out }}$ such that $\langle A\rangle$ remains at its set-point $A_{\text {set }}^{\text {out }}=2.0$. Because of the homeostatic condition $\langle A\rangle=A_{\text {set }}^{\text {out }}$, there is an inverse relationship between the (integrated) amplitude of $A$ and the frequency $\omega$ of the conservative oscillations. Consider that for a given set of rate constants the oscillator undergoes $n$ cycles during the time interval $\tau$. For each cycle, we can integrate $A$ for one period length, which results in what we may call an "integrated amplitude" $A_{1}$. Using $A_{1}$, the average of $A$ for $n$ cycles can be expressed by $\langle A\rangle=n A_{1} / \tau$. Because $n / \tau=\omega$, we get $\langle A\rangle=A_{1} \omega=A_{\text {set }}^{\text {out }}$, showing that the integrated amplitude $A_{1}$ is inversely proportional to the oscillator's frequency $\omega$. Because of the conservative character of the oscillations, random changes in $A$ and $E$ lead also to changes in the amplitudes of $A$ and $E$ as well as to the frequency, while keeping the average value of $A,\langle A\rangle$, close to $A_{\text {set }}^{\text {out }}$ (Figure 4, parts e and f).

Correspondingly, by neglecting inflow perturbation $k_{\text {pert }}^{\text {inflow }}$ and assuming zero order degradation of outflow perturbation (Michaelis-Menten dynamics with low $K_{M}$ value) in the dynamics of $A$, the rate equations for the inflow controller in Figure 2a becomes:

$$
\begin{aligned}
& \dot{A}=k_{1} E-k_{\text {pert }}^{\text {oufflow }} \\
& \dot{E}=k_{6} E-k_{8} E A
\end{aligned}
$$

which also show conservative oscillations.

While for the outflow controller (eqs 8 and 9) the trajectories move in an anticlock-wise manner, for the inflow controller (eqs 14 and 15) the trajectories move clockwise with $\langle A\rangle=A_{\text {set }}^{\text {in }}=k_{6} / k_{8}$. Homeostasis is kept as long as there is a sufficient large outflow from $A$ that the inflow controller can compensate (data not shown).

\section{LIMIT-CYCLE OSCILLATIONS}

We wondered whether it would be possible to construct limitcycle oscillations with the inflow/outflow controller motifs from Figure 2 such that the homeostatic condition $\langle A\rangle=A_{\text {set }}$ would be still obeyed. For both motifs this can be achieved by including an additional intermediate $a$ to the network. Figure 5a shows this for the inflow controller motif with the following rate equations:

$$
\begin{aligned}
& \dot{a}=k_{1} E-k_{10} a \\
& \dot{A}=k_{\text {pert }}^{\text {inflow }}+k_{10} a-k_{\text {pert }}^{\text {outflow }} A \\
& \dot{E}=k_{6} E-k_{8} A E
\end{aligned}
$$

Figure $5 \mathrm{~b}$ shows projections of the 3-dimensional limit cycle on to the $A-E$ phase plane with different initial conditions. In each of these cases $\langle A\rangle=A_{\text {set }}^{\text {in }}=k_{6} / k_{8}$ is obeyed. Figure $5 \mathrm{c}$ shows the time behavior when initial condition 3 from Figure $5 \mathrm{~b}$ is used. The controller has the typically properties of an inflow controller, i.e., breakdown of homeostasis when the uncontrollable inflow of $A$ is dominating over the outflows (Figure 5d). 
$\mathrm{a}_{3}$

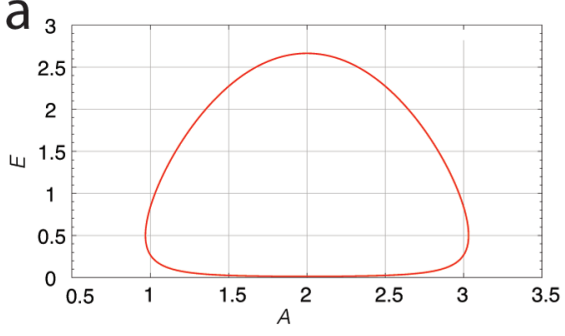

C
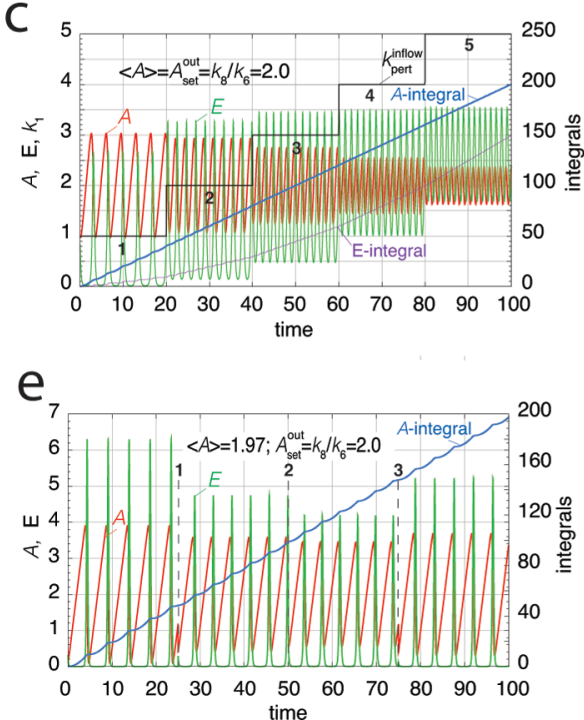

b

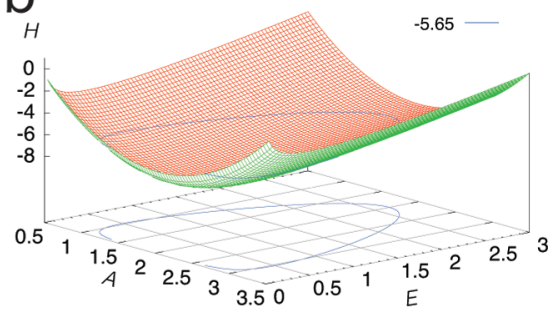

d

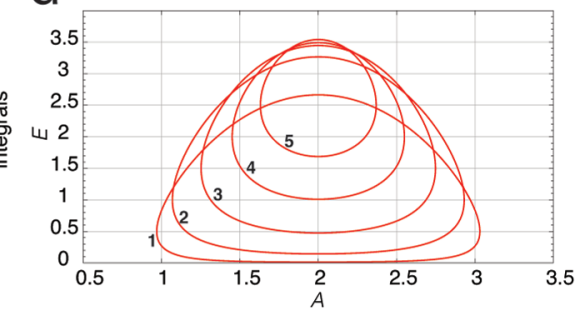

f

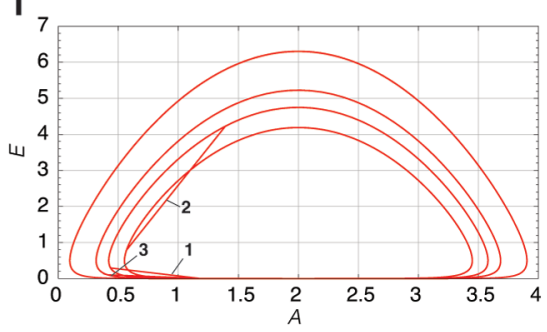

Figure 4. Conservative oscillations for the outflow controller described by eqs 6 and 7. (a) Numerical computation of closed phase orbit with rate constants (in a.u.) $k_{\text {pert }}^{\text {inflow }}=1.0, k_{3}=2.0, K_{M 4}=1 \times 10^{-6}, k_{6}=5.0, k_{8}=10.0$, and the initial concentrations $A_{0}=1.0$, and $E_{0}=0.8333$. The calculated average of $A$ (eq 13) is $\langle A\rangle=A_{\text {set }}^{\text {out }}=k_{8} / k_{6}=2.0$. The trajectory runs counterclockwise in the $A$ (abscissa) $-E$ (ordinate) phase plane. (b) Calculated $H$-function from eqs 8 and 9 taking the form $H=-k_{\text {pert }}^{\text {inflow }} \xi+k_{3} e^{\xi}+0.5 k_{6} A^{2}-k_{8} A$, where $\xi=\ln (E)$. Using the rate constants and initial conditions from part a gives a value for $H$ of -5.65 , which leads to the same closed loop trajectory (blue line) as calculated in part a. (c) The successive increase of $k_{\text {pert }}^{\text {inflow }}$ (black lines) from 1.0 to 5.0 leads to an increase in the oscillator's frequency and a decrease in the amplitude of $A$ (red lines). The blue line is the integral of $A$, i.e. the value of $(1 / t) \int_{0}^{t} A\left(t^{\prime}\right) d t^{\prime}$ for time $t$. The apparent linearity shows that $\langle A\rangle$ is constant and equal to $A_{\text {set }}^{\text {out }}$. Oscillations in $E$ are shown in green and the E-integral as a function of time is given in purple. (d) Phase behavior of the system in part c. Numbers $1-5$ indicate the trajectories for the values of $k_{\text {pert }}^{\text {inflow }}$ changing from 1.0 to 5.0 as indicated in part c. (e) Same system as in part a, but concentrations in $A$ and $E$ are changed randomly between zero and one at time units $0,25,50$, and 75 . Also here $\langle A\rangle$ is close to $A_{\text {set }}^{\text {out }}$, but due to the changes in $A$ at the transitions the global $A$-average (from $t=0$ to $t=100)$ is not precisely at $A_{\text {set }}^{\text {out }}$. However, for each of the time intervals $(0-25),(25-50),(50-75),(75-100)$ we have that $\langle A\rangle=A_{\text {set }}^{\text {out }}=2.0$. Dashed lines 1,2 , and 3 indicate the random changes made in $A$ and $E$. (f) Phase plane behavior of the system described in part e. Numbers $1-3$ relate to the (stochastic) changes made in $A$ and $E$ at time units 25,50 , and 75.

In a similar approach, limit cycle oscillations with a homeostatic outflow controller motif (Figure 2b) can be obtained (data not shown).

\section{LOTKA-VOLTERRA AND RELATED OSCILLATORS}

The Lotka-Volterra oscillator (LVO) equations

$$
\begin{aligned}
& \dot{A}=k_{1} A-k_{3} A E \\
& \dot{E}=k_{6} A E-k_{8} E
\end{aligned}
$$

have been formulated independently by Lotka and Volter$\mathrm{ra}^{34-36}$ and have been the subject of many studies especially within chemical oscillator theory, ${ }^{37,38}$ predator-prey interactions, ${ }^{39}$ as well as in economics. ${ }^{40}$ The LVO contains two autocatalytic loops and can be viewed having an outflow controller structure relative to $A$ and an inflow controller structure relative to $E$ (Figure $6 \mathrm{a}$ ). The oscillations are conservative and any perturbation in $A$ (via $k_{\text {pert }, A}^{\text {inflow }}, k_{\text {pert }, A}^{\text {outflow }}$ ) or in $E$ (via $k_{\text {pert }, E}^{\text {inflow }}, k_{\text {pert }, E}^{\text {ouflow }}$ ) will lead to a new closed trajectory in the $A-E$ phase space. The conservative nature of the LVO may be considered as unrealistic as it lacks the stability properties of limit-cycles. ${ }^{36}$ However, any perturbation in $A$ (or in $k_{1}$ or $k_{3}$ ) is counteracted such that the average value of $A,\langle A\rangle$, returns to its set-point $A_{\text {set }}^{\text {out }}=k_{8} / k_{6}$. The situation is analogous to that shown in Figures $4 \mathrm{c}-\mathrm{f}$, but with the difference that in the LVO, also perturbations in $E$ (or in $k_{6}$ or $k_{8}$ ) are compensated and $\langle E\rangle$ (as defined in eq 13) is kept at the homeostatic set-point $E_{\text {set }}^{\text {out }}=k_{1} / k_{3}$.

Interchanging $A$ and $E$ in eqs 19 and 20 leads to the negative feedback shown in Figure $6 \mathrm{~b}$, where the system has now an inflow control motif with respect to $A$ and an outflow controller motif with respect to $E$. Thus, the autocatalytic loop in $A$ controls the homeostatic behavior in $E$, while the autocatalytic loop in $E$ controls the homeostasis in $A$. The negative feedback loops described in Figure 6, parts a and b, behave differently when $A$ or $E$ are subject to perturbations in their inflow/ outflow fluxes to or from $A / E$. As the feedback in Figure 6a is an outflow type of controller with respect to $A$, any outflow perturbation $\left(k_{\text {pert }, A}^{\text {outflow }}\right)$ exceeding the (autocatalytic) inflow flux to $A$ will destroy the homeostatic behavior of the $A$-controller. Similarly, any inflow perturbation to $E\left(k_{\text {pert }, E}^{\text {inflow }}\right)$ exceeding the outflow flux mediated by $k_{8}$ will destroy the homeostasis in $E$. 
a

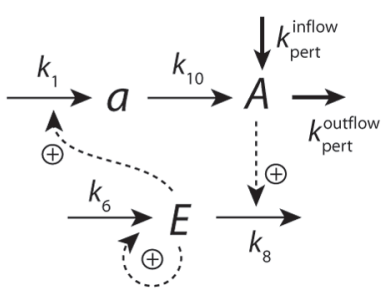

C

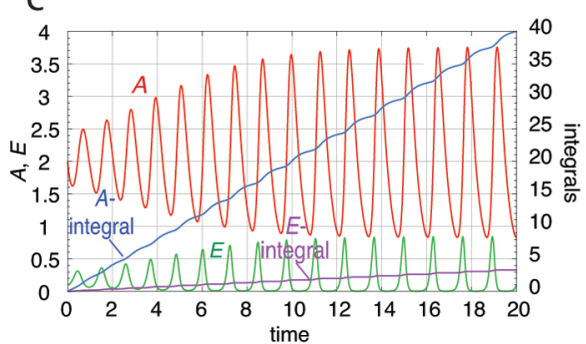

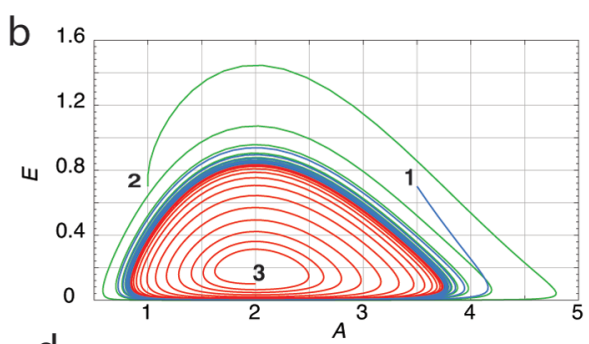

d

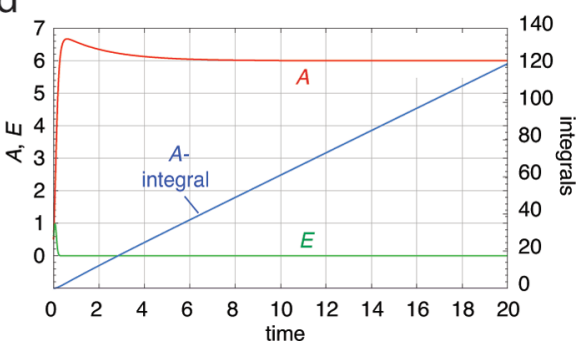

Figure 5. (a) "Extended" inflow controller showing limit-cycle oscillations. (b) Approach to limit-cycle at initial conditions: 1, $a_{0}=2.0, A_{0}=3.5, E_{0}=$ $0.7 ; 2, a_{0}=0.1, A_{0}=1.0, E_{0}=0.7 ; 3, a_{0}=0.1, A_{0}=2.0, E_{0}=0.1$. Rate constant values: $k_{\text {pert }}^{\text {inflow }}=1.0, k_{\text {pert }}^{\text {outflow }}=3.0, k_{6}=20.0, k_{8}=10.0, k_{1}=30.0, k_{10}=$ 10.0. (c) Time profile of oscillations in part b with initial conditions 3. $\langle A\rangle=A_{\text {set }}^{\text {in }}=k_{6} / k_{8}=2.0$. (d) Breakdown of homeostatic control $(\langle A\rangle=6.0)$ when $k_{\text {pert }}^{\text {inflow }}=3.0$ and $k_{\text {pert }}^{\text {oufflow }}=0.5$ leading to $k_{\text {pert }}^{\text {inflow }}>A_{\text {set }}^{\text {in }}$ pert ouflow. All other rate constants as in part $b$.

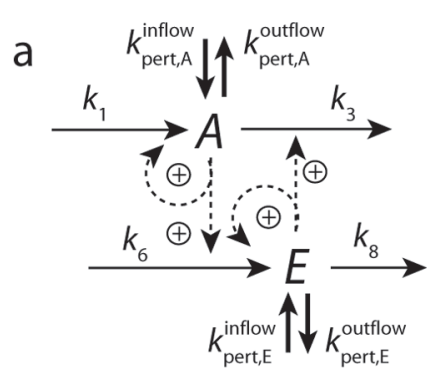

C

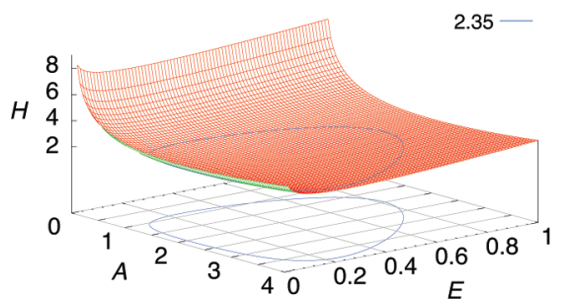

b

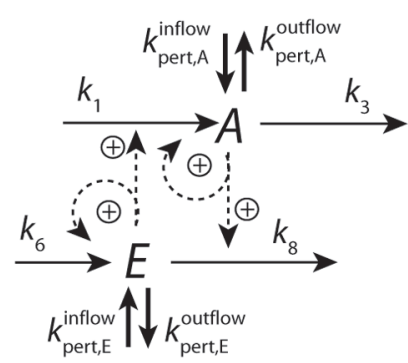

d

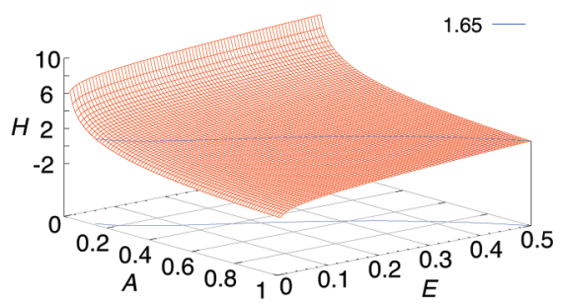

Figure 6. Lotka-Volterra oscillator. (a) The negative feedback structure defines an outflow-type of controller with respect to $A$ and an inflow-type of controller with respect to $E$. Rate constants $k_{\text {pert }, A}^{\text {inflow }}, k_{\text {pert }, A}^{\text {outflow }}, k_{\text {pert }, E}^{\text {inflew }}$ and $k_{\text {pert }, E}^{\text {outflow }}$ describe perturbative inflow and outflow fluxes. (b) The LVO with negative feedback structure defining an inflow-type of controller with respect to homeostasis in $A$, and an outflow-type of controller with respect to E. (c) The LVO from (a) with $k_{1}=1.0, k_{3}=2.0, k_{6}=1.0, k_{8}=2.0$, and $k_{\text {pert }, A}^{\text {ouflow }}=0.5$. All other rate constants are zero. Initial concentrations are $A_{0}=$ 1.0 , and $E_{0}=0.5$. The oscillations are shown as a closed orbit on the $H(A, E)$-surface with projection on to the $A-E$ phase plane with $\langle A\rangle=A_{\text {set }}^{\text {out }}=k_{8} /$ $k_{6}=2.0$. (d) Same system as in part c, but $k_{\text {pert }, A}^{\text {outlow }}=1.5$. Homeostasis in $\langle A\rangle$ is lost and the system approaches a steady state well below $A_{\text {set }}^{\text {out }}$.

In Figure $6 \mathrm{~b}$, the situation is reversed; i.e., any inflow perturbation in $A\left(k_{\text {pert }, A}^{\text {inflow }}\right)$ exceeding the outflow flux mediated by $k_{3}$ will destroy homeostasis in $A$, while any outflow perturbation from $E\left(k_{\text {pert }, E}^{\text {outflew }}\right)$ exceeding the autocatalytic inflow flux mediated by $k_{6}$ will destroy the homeostasis in $E$. We illustrate these behaviors by one example using a first-order outflow perturbation with rate constant $k_{\text {pert }, A}^{\text {ouffow }}$ from $A$ for the LVO in Figure 6a. By including the term $-k_{\text {pert }, A}^{\text {ouflow } A}$ to eq 19 (the other perturbation terms are $k_{\text {pert }, A}^{\text {inflow }}=k_{\text {per }, E}^{\text {infl }}=k_{\text {pert }, E}^{\text {ouf }}=0$ ), the system remains conservative and by using the method outlined above to calculate $H$, we get

$$
H(A, E)=\left(k_{\text {pert }, A}^{\text {outflow }}-k_{1}\right) \ln (E)+k_{3} E+k_{6} A-k_{8} \ln (A)
$$

As long as $k_{\text {pert, } A}^{\text {outflow }}<k_{1}$, homeostasis in $\langle A\rangle$ is maintained, and the system shows oscillations. The (oscillatory/closed) trajectory on the $H$-surface is shown in Figure 6c. However, when $k_{\text {pert, } A}^{\text {outflow }}>k_{1}$, homeostasis breaks down and the steady state value in $A$ settles below its homeostatic set-point. The trajectory on the $H$-surface is shown in Figure $6 \mathrm{~d}$.

By including an additional intermediate, the LVO schemes in Figure 6, parts a and $b$, can be transformed into limit-cycle oscillations. We show here the results for the "inflow-controller version" with respect to $A$ (Figure 7a). Species $a$, which is induced by the autocatalytically formed $E$ is a precursor to $A$ on which $A$ itself is formed autocatalytically. $A$ on its side induces the removal/degradation of $E$ causing a negative feedback necessary to get oscillations and homeostasis. Limit-cycle 


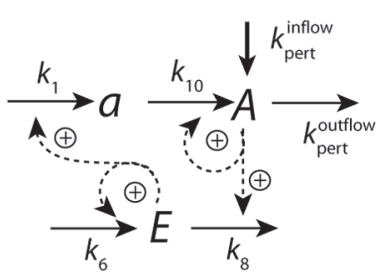

C

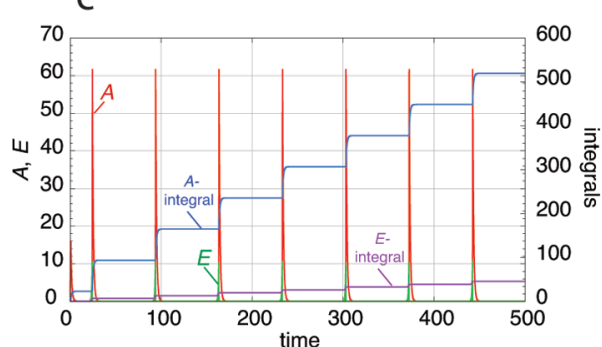

b

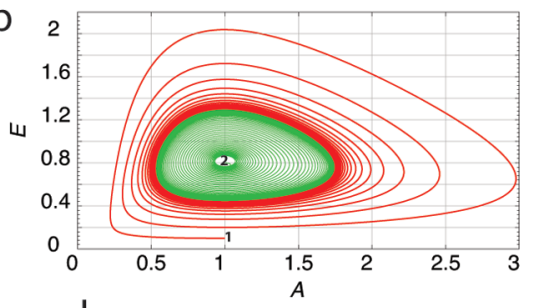

d

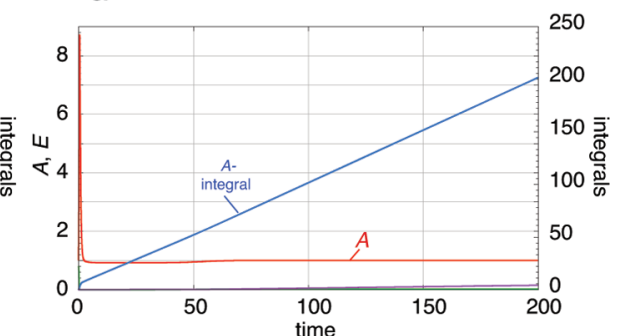

Figure 7. (a) Extension of the LVO from Figure $6 \mathrm{~b}$ with variable inflow and outflow perturbations ( $k_{\text {pert }}^{\text {inflow }}$ and $\left.k_{\text {pert }}^{\text {oufflow }}\right)$ in $A$. (b) Demonstration of limit-cycle behavior. Rate constants: $k_{1}=11.0, k_{6}=2.0, k_{8}=2.0, k_{10}=0.5, k_{\text {pert }}^{\text {inflow }}=1.0$ and $k_{\text {pert }}^{\text {ouflow }}=10.0$. Initial concentrations for 1: $a_{0}=10.1, A_{0}=$ 1.0, $E_{0}=0.1$ and for 2: $a_{0}=18.0, A_{0}=1.0, E_{0}=0.8$. The homeostatic set point for $A$ is $A_{s e t}^{i n}=k_{6} / k_{8}=1.0$, and confirmed by calculating $\langle A\rangle$. (c) Pulsatile oscillations with period $P=69.7$ time units. Rate constants as in part $\mathrm{b}$ except $k_{\text {pert }}^{\text {inflow }}=1 \times 10^{-6}$ and $k_{\text {pert }}^{\text {oufflow }}=1.0$. Initial concentrations $a_{0}=$ 20.0, $A_{0}=1.0, E_{0}=0.8$. Although $A$ peak-values are above $60 \mathrm{au}$, the determined average of $A$ is $\langle A\rangle=1.04$ and very close to $A_{\text {set }}^{\text {in }}=1.0$. (d) Nonoscillatory homeostasis of $A$. Rate constants as in (b) except $k_{\text {pert }}^{\text {inflow }}=3.5$ and $k_{\text {pert }}^{\text {outlow }}=3.8$. Initial concentrations as in part $\mathrm{c}$.

oscillations can be demonstrated (Figure $7 \mathrm{~b}$ ) for a variety of rate constant values. Dependent on the rate constant values the period length can vary considerably ranging from about 70 time units (Figure $7 \mathrm{c}$ ) to a fraction of a time unit. Because of the inflow-type of controller relative to $A$, homeostasis in $A$ is observed as long as the environmental perturbative zero-order inflow flux $k_{\text {pert }, A}^{\text {inflow }}$ to $A$ is smaller than the perturbative outflow flux from $A$ determined by $k_{\text {pert }, A}^{\text {oufflow }}$. Homeostasis in $A$ can be observed both in oscillatory/pulsatile mode (Figure 7c) or in nonoscillatory mode (Figure $7 \mathrm{~d}$ ).

\section{DISCUSSION}

We have shown that autocatalysis/positive feedback is an alternative way to introduce integral control in homeostatic controller motifs, while the inflow/outflow properties ${ }^{21}$ of the motifs remain preserved. We have demonstrated this for two controller motifs, but the method can be applied for other twocomponent controller motifs that were recently identified. ${ }^{28}$

An interesting aspect when comparing integral control between the zero-order kinetic (Michaelis-Menten) approach (Figure 1) and the here described autocatalytic method (Figure 2 ) is related to the accuracy of the controller. In the zero-order approach the $K_{M}$ value of the process removing the controlled variable $E$ serves as a measure for controller accuracy of the two controllers addressed here. At low $K_{M}$ values the controller accuracy is high, i.e., $A$ is close to $A_{\text {set }}$, while at high $K_{M}$ values the accuracy of the controller is low, ${ }^{28}$ and the steady state of $A$ is dependent upon the type of the controller. For the inflow controller, a high $K_{M}$ will lead to a steady state in $A$ which will be higher than $A_{\text {set }}$ (as indicated by the equation for $\dot{E}$ in Figure $1 \mathrm{~b}$ ), while in the outflow controller (Figure 1c) a high $K_{M}$ will lead to a $A$ steady state, which has a lower value than $A_{\text {set }}$. In the autocatalytic approach the controller accuracy is intrinsically perfect, because no requirements such as $K_{M 1} / K_{M 3} \ll E$ (Figure $1)$ are necessary. While we considered a first-order reaction in the $A$-mediated removal of $E$ (Figure 2), the kinetics could also be of Michaelis-Menten type or any other nonzero reactionorder with respect to $A$, as long as the reaction-order with respect to $E$ for its autocatalytic formation and for its degradation remains the same.

In this study we have focused on the two homeostatic inflow and outflow controller motifs (Figure 1/Figure 2) with a close relationship to the LVO and similar oscillators (Figure 6). In 1925, Lotka described his attempts to explain biological homeostatic behavior on the basis of Le Chatelier's principle. ${ }^{8}$ He concluded negatively, and ironically, it seems that he was unaware that the equations that bear today his name have homeostatic properties.

The LVO and derivative models are well-known for their usage in ecological systems, ${ }^{36,39}$ which have generally been thought of as homeostatic systems. ${ }^{41}$ While the conservative nature of the LVO is mostly considered to be a drawback when considering system stability we have shown that they already contain, due to their autocatalytic nature homeostatic behavior in $\langle A\rangle$ or $\langle E\rangle$ (Figure 4), which can be extended to limit-cycle models (Figures 5 and 7).

There is an extensive literature showing that autocatalysis/ positive feedback in combination with negative feedback loops can be the source for a variety of dynamic behaviors including excitability, $^{42,43}$ oscillations, ${ }^{38,44-46}$ spatial pulse propagation, ${ }^{38,43}$ bi- or multistability, ${ }^{38,47,48}$ as well as Turing structures. $^{49}$ While homeostasis is generally associated with negative feedback regulation, ${ }^{21,50,51}$ combinations of positive and negative feedback loops with respect to homeostasis have also been addressed. An example is the hypothalamus-pituitaryadrenal system, ${ }^{52}$ where the positive feedback is considered to be a crucial component to self-stabilize the system. A related behavior has been observed earlier by Cinquin and Demongeot, ${ }^{53}$ showing that a certain strength of the positive feedback in a combined positive-negative feedback model is required to obtain stability of the system. Maintenance of stem cell homeostasis in the apical meristem in rice has recently been reported to be due to a positive autoregulation of the KNOX gene. $^{54}$

Calcium is an important signaling molecule in all living cells and its concentration is tightly regulated in the cytosol and 
organelles. ${ }^{55}$ For example, disregulation of Ca-homeostasis is involved in various neurodegenerative diseases. ${ }^{56}$ Both positive and negative feedback loops have been identified in cellular Caregulation showing behaviors such as sparks, waves, bursts or oscillations. ${ }^{57-59}$ The observed positive feedback loops in calcium regulation may be part of the homeostatic mechanisms that maintain cytosolic and organellar calcium levels, ${ }^{55}$ but little is presently known in this respect.

For certain neurons, iron uptake has been found to occur by an oxidative-stress mediated positive feedback loop. ${ }^{60}$ The role of the positive feedback is still unclear, but also here it could be that the positive feedback participates in the regulation of iron by possibly participating in the determination of the iron homeostatic set-point.

The notion that autocatalysis (or positive feedback) is a source of robust stability may appear counterintuitive. However, it should be kept in mind that the autocatalytic loop generating $E$ (or $A$ ) is part of an overall negative feedback loop (controller motif). ${ }^{21,28}$ Positive feedback is an important driving force for growth and development, ${ }^{61}$ but needs to be limited by negative feedback to avoid runaway states. ${ }^{41}$

\section{CONCLUSION}

We have shown that autocatalysis/positive feedback can be a mechanism leading to integral control and thereby resulting into robust homeostatic and adaptive behaviors. However, as indicated by the biological examples above, we presently still know little about how positive feedback loops are involved in the cellular organization of homeostatic behavior.

\section{AUTHOR INFORMATION}

\section{Corresponding Author}

*Telephone: (47) 5183-1887. Fax: (47) 5183-1750. E-mail: peter.ruoff@uis.no.

\section{Notes}

The authors declare no competing financial interest.

\section{ACKNOWLEDGMENTS}

This work was supported in part by grants from the Norwegian Research Council to X.Y.N. (183085/S10) and I.W.J. (167087/ V40).

\section{REFERENCES}

(1) Cannon, W. B. The Wisdom of the Body. Revised and Enlarged ed.; Norton: New York, 1939.

(2) Cannon, W. B. Physiol. Rev. 1929, 9, 399-431.

(3) Langley, L. L., Ed. Homeostasis. Origins of the Concept; Dowden, Hutchinson \& Ross, Inc.: Stroudsburg, PA, 1973.

(4) Moore-Ede, M. C. Am. J. Physiol. 1986, 250, R737-R752.

(5) Mrosovsky, N. Rheostasis. The Physiology of Change; Oxford University Press: New York, 1990.

(6) Sterling, P.; Eyer, J. In Handbook of Life Stress, Cognition and Health; Fisher, S., Reason, J., Eds.; John Wiley \& Sons: New York, 1988; pages 629-649.

(7) Schulkin, J. Rethinking Homeostasis. Allostatic Regulation in Physiology and Pathophysiology; MIT Press: Cambridge, MA, 2003.

(8) Lotka, A. J. Elements of Physical Biology; Williams \& Wilkins Company: Baltimore, MD, 1925.

(9) Castellan, G. W. Physical Chemistry, 3rd ed.; Addison-Wesley: Reading, MA, 1983.

(10) von Bertalanffy, L. Perspectives on General System Theory; George Braziller: New York, 1975.

(11) Savageau, M. A. Biochemical Systems Analysis. A Study of Function and Design in Molecular Biology; Addison-Wesley: Reading, MA, 1976.
(12) Heinrich, R.; Schuster, S. The Regulation of Cellular Systems; Chapman and Hall: New York, 1996.

(13) Hughes, G. M. Homeostasis and Feedback Mechanisms; Academic Press: New York, 1964.

(14) Milsum, J. H. Biological Control Systems Analysis; McGraw-Hill: New York, 1966.

(15) Saunders, P.; Koeslag, J.; Wessels, J. J. Theor. Biol. 1998, 194, 163-173.

(16) Yi, T. M.; Huang, Y.; Simon, M. I.; Doyle, J. Proc. Natl. Acad. Sci. U.S.A. 2000, 97, 4649-4653.

(17) Milo, R.; Shen-Orr, S.; Itzkovitz, S.; Kashtan, N.; Chklovskii, D.; Alon, U. Science 2002, 298, 824-827.

(18) Yang, Q.; Lindahl, P. A.; Morgan, J. J. J. Theor. Biol. 2003, 222, 407-423.

(19) Drengstig, T.; Ueda, H. R.; Ruoff, P. J. Phys. Chem. B 2008, 112, $16752-16758$.

(20) Alon, U. An Introduction to Systems Biology: Design Principles of Biological Circuits; Chapman \& Hall: New York, 2006.

(21) Ni, X. Y.; Drengstig, T.; Ruoff, P. Biophys. J. 2009, 97, 12441253.

(22) Ma, W.; Trusina, A.; El-Samad, H.; Lim, W. A.; Tang, C. Cell 2009, 138, 760-773.

(23) Ang, J.; Sangram, B.; Ingalls, B. P.; McMillen, D. R. J. Theor. Biol. 2010, 266, 723-738.

(24) Ruoff, P.; Zakhartsev, M.; Westerhoff, H. V. FEBS J 2007, 274, 940-950.

(25) Segel, L. A.; Goldbeter, A.; Devreotes, P. N.; Knox, B. E. J. Theor. Biol. 1986, 120, 151-179.

(26) Warwick, K. An Introduction to Control Systems. 2nd ed.; World Scientific: Singapore, 1996.

(27) El-Samad, H.; Goff, J. P.; Khammash, M. J. Theor. Biol. 2002, $214,17-29$.

(28) Drengstig, T.; Jolma, I. W.; Ni, X. Y.; Thorsen, K.; Xu, X. M.; Ruoff, P. Biophys. J. 2012, submitted.

(29) Huang, Y.; Drengstig, T.; Ruoff, P. Plant, Cell Environ. 2012, 35, 917-928.

(30) Radhakrishnan, K.; Hindmarsh, A. C. Description and Use of LSODE, the Livermore Solver for Ordinary Differential Equations. NASA Reference Publication 1327, Lawrence Livermore National Laboratory Report UCRL-ID-113855; National Aeronautics and Space Administration, Lewis Research Center: Cleveland, OH, 1993.

(31) Arnold, D.; C, P. J. Ordinary Differential Equations using MATLAB; Prentice Hall: New York, 1999.

(32) Press, W. H.; Flannery, B. P.; Teukolsky, S. A.; Vetterling, W. T. Numerical Recipes. The Art of Scientific Computing (Fortran Version); Cambridge University Press: Cambridge, U.K., 1986.

(33) Nicolis, G.; Prigogine, I. Self-Organization in Nonequilibrium Systems; John Wiley \& Sons: New York, 1977.

(34) Lotka, A. J. J. Phys. Chem. 1910, 14, 271-274.

(35) Lotka, A. J. J. Am. Chem. Soc. 1920, 42, 1595-1599.

(36) Murray, J. D. Mathematical Biology. I. An Introduction. Third ed.; Springer-Verlag: Berlin, 2002.

(37) Higgins, J. Ind. Eng. Chem. 1967, 59, 18-62.

(38) Field, R. J.; Burger, M. Oscillations and Traveling Waves in Chemical Systems; John Wiley \& Sons: New York, 1985.

(39) Wangersky, P. J. Annu. Rev. Ecol. Syst. 1978, 9, 189-218.

(40) Goodwin, R. M. In Socialism, Capitalism and Economic Growth. Essays presented to Maurice Dobb; Feinstein, C. H., Ed.; Cambridge University Press: Cambridge, U.K., 1967; pp 54-58.

(41) DeAngelis, D. L.; Post, W. M.; Travis, C. C. Positive Feedback in Natural Systems; Springer-Verlag: Berlin, 1986.

(42) Markin, V. S.; Pastushenko, V. F.; Chizmadzhev, Y. A. Theory of Excitable Media; John Wiley \& Sons: New York, 1987.

(43) Winfree, A. T. The Geometry of Biological Time. 2nd ed.; Springer-Verlag: New York, 2000.

(44) Eiswirth, M.; Freund, A.; Ross, J. Adv. Chem. Phys. 1991, LXXX, 127-199.

(45) Goldbeter, A. Biochemical Oscillations and Cellular Rhythms; Cambridge University Press: Cambridge, U.K., 1996. 
(46) Novak, B.; Tyson, J. J. Nat. Rev. Mol. Cell Biol. 2008, 9, 981991.

(47) Crowley, M. F.; Epstein, I. R. J. Phys. Chem. 1989, 93, 24962502.

(48) Pomerening, J. R. Curr. Opin. Biotechnol. 2008, 19, 381-388.

(49) Lengyel, I.; Epstein, I. R. Acc. Chem. Res. 1993, 26, 235-240.

(50) Tyson, J. J.; Chen, K. C.; Novak, B. Curr. Opin. Cell Biol. 2003, $15,221-231$.

(51) Sneppen, K.; Krishna, S.; S., S. Annu. Rev. Biophys. 2010, 39, 43-59.

(52) Conrad, M.; Hubold, C.; Fischer, B.; Peters, A. J. Biol. Phys. 2009, 35, 149-162.

(53) Cinquin, O.; Demongeot, J. C. R. Biologies 2002, 325, 10851095.

(54) Tsuda, K.; Ito, Y.; Sato, Y.; Kurata, N. Plant Cell 2011, 23, $4368-4381$.

(55) Stael, S.; Wurzinger, B.; Mair, A.; Mehlmer, N.; Vothknecht, U. C.; Teige, M. J. Exp. Bot. 2012, 63, 1525-1542.

(56) Wojda, U.; Salinska, E.; Kuznicki, J. IUBMB Life 2008, 60, 5755902.

(57) Larter, R.; Craig, M. G. Chaos 2005, 15, 047511.

(58) Politi, A.; Gaspers, L. D.; Thomas, A. P.; Höfer, T. Biophys. J. 2006, 90, 3120-3133.

(59) Cheng, H.; Lederer, W. J. Physiol. Rev. 2008, 88, 1491-1545.

(60) Núñez-Millacura, C.; Tapia, V.; Muñoz, P.; Maccioni, R. B.; Núñez, M. T. J. Neurochemistry 2002, 82, 240-248.

(61) Takeda, S.; Gapper, C.; Kaya, H.; Bell, E.; Kuchitsu, K.; Dolan, L. Science 2008, 319, 1241-1244. 